\title{
Use of MRI to Measure Whole Brain Atrophy in MS Patients
}

\author{
P. MazGaj ${ }^{a}, *$ Z. DrzazGa ${ }^{a}$, I. Karpiel ${ }^{a}$, A. Giec-Lorenz ${ }^{b}$ And E. KrzystaneK ${ }^{c}$ \\ ${ }^{a}$ Department of Medical Physics, Institute of Physics, University of Silesia, \\ Uniwersytecka 4, 40-007 Katowice, Poland \\ ${ }^{b}$ Helimed Diagnostic Imaging Sp. z o.o., Laboratory of Magnetic Resonance Imaging, \\ Medyków 14, 40-752 Katowice, Poland \\ ${ }^{c}$ Department of Neurology, Medical University of Silesia in Katowice, ul. Medyków 14, 47-750 Katowice, Poland
}

\begin{abstract}
Nowadays magnetic resonance imaging is used for anatomical assessment of human brain structures in neurodegenerative disorders causing brain atrophy for instance in multiple sclerosis (MS) or in Alzheimer Disease. Pathological brain tissue loss can be described in terms of change in the brain parenchymal fraction (BPF). This work shows the impact of segmentation method in SPM12 and additional segmentation in Computational Anatomy Toolbox (CAT12) on calculated BPF value for patients suffer from with MS no treated and treated with disease-modifying drug (DMD) interferon-beta (INFb) for one year and two years. Both methods confirm that brain parenchymal fraction decreases with age, nevertheless for patients not treated INFb decreases faster than for treated. An usability of Lesion Segmentation Tool toolbox in process of automatic detection and segmentation $T_{2}$ hyperintense lesions in FLAIR images is discussed.
\end{abstract}

DOI: 10.12693/APhysPolA.133.725

PACS/topics: 87.57.Nk, 87 .61.Pk, 87.66.Uv

\section{Introduction}

Presently multiple sclerosis (MS) is one of the most common demyelinating diseases of central nervous system (CNS), which progressively reduce Patients quality of life by a gradual loss of brain tissue. The beginning of MS is often unexpected. The most common first symptoms of the disease are i.e. paresthesias, impaired coordination, visual impairment or weakness of muscles $[1,2]$. This chronic, inflammatory disease can occur in four types, which differ in the course of the disease: secondary progressive MS, primary progressive MS, progressive-relapsing MS and the most common form relapsing-remitting MS [2]. To slow down the progression of the disease, drug therapies currently used which the main purpose are speeding recovery after MS relapse, slowing the progression of the disease and managing symptoms [3].

According to the data presented by the European Multiple Sclerosis Platform (EMSP) in Poland living at least 45,000 people with MS (population 38.5 million) [4]. It mainly concerns young people and becomes a social problem requiring early diagnosis and treatment.

One of the diagnostic methods which revolutionised the diagnosis of MS is magnetic resonance imaging (MRI). Depending on the applied MRI sequences we have ability to image demyelinating plaques as: hypointense lesions and hyperintense lesions in anatomic $T_{1}$ images and diffusion - weighted imaging (DWI), respectively [5]. The Apparent Diffusion Coefficient Maps (ADC) are also useful for the quantitative assessment of pathological states

*corresponding author; e-mail: patrycjamazgaj@gmail.com of the brain, characterisation of lesions [6]. Moreover, application of Gadolinium-based contrast agent allows find active lesions in $T_{1}$ sequence [5].

Diagnostic of MS is focused on changes in white matter (WM), which are the most recognizable and specific for these disease unit. However, in multiple sclerosis grey matter is also destroyed but changes of cortical lesions are harder to detect. Recently it was reported, it is possible to imaging grey matter (GM) plaques using Double Inversion Recovery sequence (DIR) which supressing signal from white matter and cerebrospinal fluid (CSF) increases to a large degree intracortical lesion detection compared to lesion identification on $T_{2}$ and FLAIR sequence $[7,8]$.

Magnetic resonance imaging is useful not only in the anatomical evaluation of human brain structures in neurodegenerative disorders of the CNS but also in the quantitative assessment of pathological brain tissue loss described by brain parenchymal fraction (BPF). BPF is defined as ratio between brain parenchymal volume (BPV) and intracranial volume (ICV), where BPV is a sum of WM and GM, while ICV is as sum of BPV and cerebrospinal fluid (CSF). Brain parenchymal fraction can be specified using different programs such as SPM, VBM, SIENAX and others but they can give different BPF values $[9,10]$.

In brain MRI, segmentation of brain tissues is an important step for numerical application. In this work we investigate an influence of segmentation method used in SPM12 and additional segmentation in Computational Anatomy Toolbox (CAT12) on calculation of BPF. Moreover usability of Lesion Segmentation Tool (LST) toolbox for SPM in process of automatic detection and segmentation $T_{2}$ hyperintense lesions in FLAIR images is discussed. 


\section{Materials and Methods}

\subsection{Experimental}

The retrospective analysis of MRI brain images were performed on 34 patients with diagnosed multiple sclerosis (ICD-10, G35). Patients were divided into two groups. The first consist of 25 females of age ranging from 25-59 and 9 females of age ranging from 22-44 treated with disease-modifying drug (DMD) interferon-beta (INFb) for one year and two years. Brain images were registered with 1,5 T MAGNETOM Aera scanner (Siemens, Erlangen, Germany) and 1.5 T GE scanner Signa HDx in Helimed Diagnostic Imaging Center for not-treated patients and for treated with INFb, respectively. The studies were performed as part of normal clinical work using protocol adapted to imaging MS containing sequences i.e. FLAIR and $T_{1}$.

\subsection{Methods of BPF determination}

To calculate volumes of white matter, grey matter and cerebrospinal fluid segmentation of MRI images must be performed. For this purpose SPM12 (Statistical Parametric Mapping, Institute of Neurology, London) in MATLAB (MathWorks, Inc.) environment and an extension to SPM package - CAT12 (C. Gaser, Structural Brain Mapping group, Jena University Hospital, Jena, Germany) which provide voxel-based morphometry (VBM) were used. Brain parenchymal and intracranial volumes for SPM12 were estimated using script [11], while in CAT12 function 'Estimate TIV' was used.

\subsection{MS Lesion segmentation tool}

Demyelinating hyperintense $T_{2}$ lesions were segmented by the lesion growth algorithm (LGA) [12] as implemented in LST toolbox version 2.0.11 (http://www.applied-statistics.de/lst.html) for SPM12 package in MATLAB. The algorithm first segmented the $T_{1}$ - weighted images into the three main tissue classes (WM, GM and CSF). Then this information was combined with coregistered FLAIR intensities in order to calculate lesion belief maps. Threshold value $(\kappa=0.1)$ was chosen for LGA [8].

\subsection{Statistics}

Analysis of variance (ANOVA) was used to examine the variance of a dependent variable and Pearson correlation tests were used to determine correlations between the variables. The significance level was set to $p<0.05$.

\section{Results and discussion}

Demyelinating process in multiple sclerosis is characterized by disintegration of myelin sheaths. The most common location of plaques is white matter of both hemispheres of the brain, mainly periventricular, ventricles, juxtacortical (see Fig. 1) and corpus callosum and temporal lobe are also involvement depending on the morphology of demyelinating lesions [1].

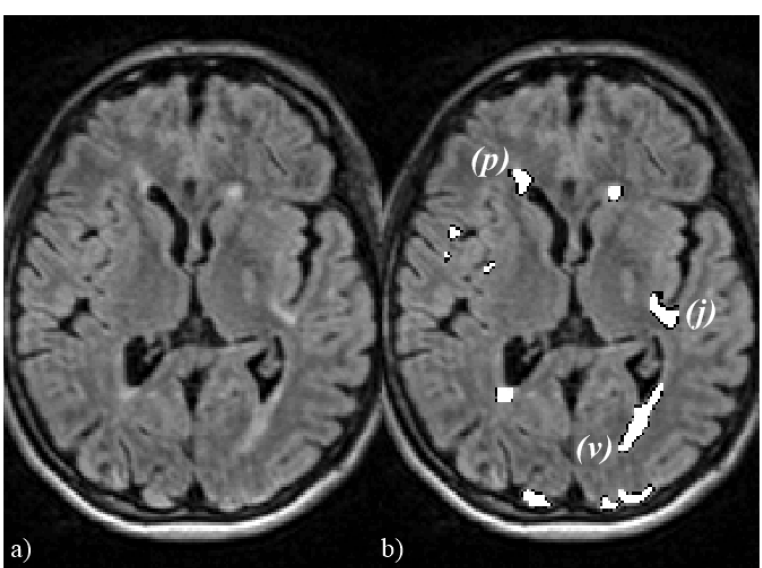

Fig. 1. a) The original FLAIR image. b) Overlayed lesion probability map $(\kappa=0.1)$ on FLAIR image highlighting white matter lesions: adjacent to the ventricles $(v)$, periventricular $(p)$ and juxtacortical $(j)$.

TABLE I

The number and volume of demyelinating white matter plaques for representative patients not treated with interferon-beta calculated in LST for initial threshold $(\kappa=0.1)$ and default vaule of MRF (1) and maximum iterations (50).

\begin{tabular}{|c|c|c|c|c|c|}
\hline \multirow{2}{*}{ ID } & \multirow{2}{*}{ Age [y] } & \multirow{2}{*}{ WM [ml] } & Number & $\mathrm{WM}[\mathrm{ml}]$ & \multirow{2}{*}{$\%$ of WM } \\
\hline & & & \multicolumn{2}{|c|}{ of plaques } & \\
\hline 1 & 25 & 482.47 & 5 & 0.81921 & 0.17 \\
\hline 2 & 31 & 561.83 & 6 & 0.39101 & 0.07 \\
\hline 3 & 34 & 612.76 & 65 & 9.16670 & 1.50 \\
\hline 4 & 37 & 455.57 & 17 & 4.66160 & 1.02 \\
\hline 5 & 45 & 435.86 & 6 & 0.25558 & 0.06 \\
\hline 6 & 46 & 453.91 & 25 & 0.90027 & 0.20 \\
\hline 7 & 46 & 487.38 & 12 & 0.81348 & 0.17 \\
\hline 8 & 49 & 437.08 & 30 & 2.6493 & 0.61 \\
\hline
\end{tabular}

Figure 2. shows the BPF of MS patients as a function of age. The linear regression confirms of brain atrophy with increasing age according to expectation. It is noteworthy the negative moderate Pearson correlation in CAT12 and SPM12 were obtained $(r=-0.41)$. For FLAIR sequence Person's correlation for untreated patients is also negative moderate $(r=-0.45)$. For patients treated with interferon-beta this correlation is weaker (after one year $r=-0.36$ and two years $r=-0.28$ ), probably due to less numerous group. The linear fits for patients treated with INFb for one year as well as two years (Fig. 2b.) are nearly the same and show only slightly smaller slope in comparison with patients not treated and also difference between them are not statistically significant. These fits are within the limits of error which is very big because of little number of patients as well as large inter-individual variation among them.

In order to make the SM diagnosis easier, LST calculating the amount and volume of demyelinating changes 


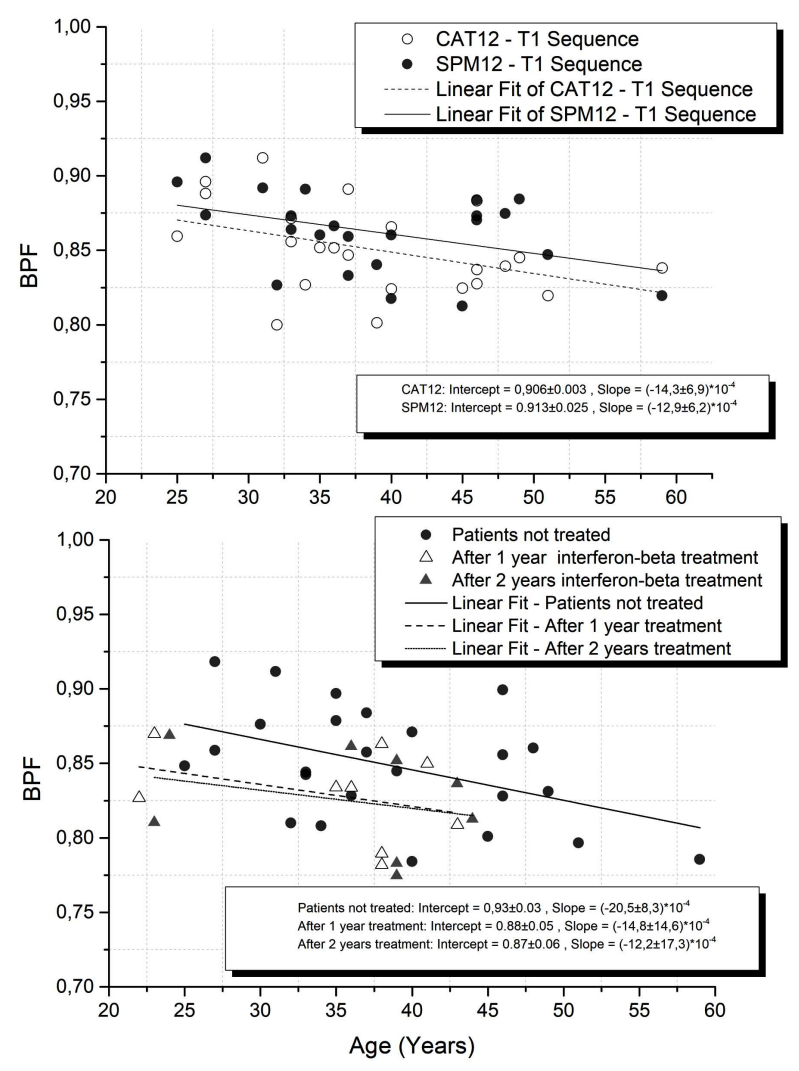

Fig. 2. BPF values in relation to age with linear fit of data. a) BPF calculated for $T_{1}$-weighted sequence for two toolboxes CAT12 and SPM12 for untreated MS patients. b) BPF calculated for FLAIR sequence in SPM12 for patients not treated and treated interferonbeta for one year and two years, respectively.

can be applied. Sample calculations for MS patients are presented in Table I.

Basing on the data in Table I, one can see that volume of white matter plaques constitute a low percentage of white matter. Nevertheless, they lead to gradual deterioration of quality of life including disability.

It is noteworthy that lesion probability map on FLAIR image (Fig. 1) highlighted demyelinating changes. Automatic lesion segmentation greatly simplifies work, but it is essential to draw attention to appropriate selection of parameters i.e. initial thresholds $(\kappa)$. Choosing a low $\kappa$-value we get a small number of white matter plaques while for the high $\kappa$ we can get result with large number of lesion, but some of them could be artefacts.

\section{Final remarks}

Magnetic resonance studies of multiple sclerosis patients are important part of diagnostic of this disease. Based on MRI data it is possible to specify additional quantitative parameters, which increases diagnostics of MS and monitoring of applied therapy.
It should be noted that brain parenchymal fraction allows for quantitative estimation of brain atrophy. Generally decrease of BPF with age in patients suffer from MS is higher in comparison with healthy individuals [13,9]. Unfortunately value of BPF depends on use of calculation method. Therefore, it is necessary to develop gold standard of BPF determination to provide a proper comparison and discussion regarding brain atrophy.

Lesion Segmentation Tool seems to be very useful in diagnostic of disease progress because allows for calculating the number and volume of demyelinating plaques but, it is essential to draw attention to appropriate selection of parameters i.e. initial thresholds $(\kappa)$.

Furthermore, it is shown that use of DMD such as interferon-beta in multiple sclerosis treatment slightly inhibits disease progression, however studies on larger research group is needed.

\section{References}

[1] P. Calabresi, Am. Farm. Physician.70, 1935 (2004).

[2] M.M. Goldenberg, Pharm. Ther. 37, 175 (2012).

[3] W. Kozubski, Neurologia Kompendium, PZWL, Warszawa 2014 (in Polish).

[4] Multiple sclerosis in Europe.

[5] B. Bodini, C. Louapre, B. Stankoff, Presse Med. 44, e159 (2015).

[6] A. Klimas, Z. Drzazga, E. Kluczewska, M. Hartel, Clin. Imag. 37, 637 (2013).

[7] O. Ciccarelli, J.T. Chen, Neurology. 78, 296 (2012).

[8] P. Kolber, S. Montag, V. Fleischer, F. Luessi, J. Wilting, J. Gawehn, A. Gröger, F. Zipp, J Nurol. 262, 1473 (2015)

[9] M. Vågberg, G. Granåsen, A. Svenningsson, PLoS One. 12; e0170018 (2017).

[10] M. Vågberg, T. Lindqvist, K. Ambarki, J.B. Warntjes, P. Sundström, R. Birgander, A. Svenningsson, AJNR Am. J. Neuroradiol. 34, 498 (2012).

[11] Ged Ridgway software get_totals.m, University College London.

[12] P. Schmidt, C. Gaser, M. Arsic, D. Buck, A. Förschler, A. Berthele, M. Hoshi, R. Ilg, V.J Schmidt, C. Zimmer, B. Hemmer, M. Mühlau, Neuroimage 59, 3774 (2012).

[13] R.A. Rudick, E. Fisher, J.-C. Lee, J. Simon, L. Jacobs and the Multiple Sclerosis Collaborative Research Group, Neurology. 53, 1698 (1999). 